\title{
REFERENCES
}

1. W. Ambrose, Structure theorem for a special class of Banach algebras, Trans. Amer. Math. Soc. vol. 57 (1945) pp. 364-386.

2. N. Jacobson, The radical and semi-simplicity for arbitrary rings, Amer. J. Math. vol. 67 (1945) pp. 300-320.

3. I. Kaplansky, Dual rings, Ann. of Math. vol. 49 (1948) pp. 689-701.

4. L. H. Loomis, An introduction to harmonic analysis, New York, Van Nostrand, 1953, pp. 100-106.

5. P. P. Saworotnow, On a generalization of the notion of $H^{*}$-algebra, Proc. Amer. Math. Soc. vol. 8 (1957) pp. 49-55.

6. M. F. Smiley, Right $H^{*}$-algebra, Proc. Amer. Math. Soc. vol. 4 (1953) pp. 1-4 Catholic University of America

\section{A BOUNDARY LAYER PROBLEM FOR AN ELLIPTIC EQUATION IN THE NEIGHBORHOOD OF A SINGULAR POINT ${ }^{1}$}

VICTOR J. MIZEL

We consider the first boundary value problem for

$$
L u=\epsilon \Delta u+A(x, y) u_{x}+B(x, y) u_{y}+C(x, y) u=D(x, y)
$$

on a region $R$ under the following hypotheses

I. $R$ is an open simply- or multiply-connected region in the $(x, y)$ plane whose boundary $S$ consists of a finite number of simple closed curves, and $R+S$ is contained in an open connected region $R_{0}$ throughout which $A(x, y), B(x, y), C(x, y)$, and $D(x, y)$ are of class $C^{6}$.

II. Along each closed curve of $S$ the functions giving $x, y$, and the boundary value $\bar{u}$ in terms of arclength are of class $C^{6}$.

III. $C(x, y)<0$ on $R_{0}$.

IV. The system (for characteristics of the abridged $(\epsilon=0)$ equation)

$$
\frac{d x}{d t}=-A(x, y), \quad \frac{d y}{d t}=-B(x, y)
$$

has as its singularities on $R+S$ a finite number of stable attractors $P_{1}, \cdots, P_{n}$.

Received by the editors February 14, 1956.

1 The author wishes at this point to express his gratitude to Professor N. Levinson who originally suggested the problem to him and who gave him encouragement throughout. 
We shall prove that if $u(x, y, \epsilon)$ is the solution to our boundary value problem (existence for small $\epsilon>0$ follows from results of Lichtenstein [4]), and if $U(x, y)$ is that solution to the abridged equation

$$
L^{0} U=A(x, y) U_{x}+B(x, y) U_{y}+C(x, y) U=D(x, y)
$$

which solves the initial value problem for $R+S-P_{1}-\cdots-P_{n}$ : $U=\bar{u}$ on those portions of $S$ where the solutions of (1) cross into $R$, then throughout $R-P_{1}-\cdots-P_{n}, v(x, y, \epsilon) \equiv U(x, y)-u(x, y, \epsilon)$ approaches zero as $\epsilon \rightarrow+0$ except possibly, as will be seen from the proof, at characteristics of (1) which are somewhere tangent to $S$.

Now Levinson [3] has proved this in the case where (1) has no singularities on $R$. Indeed his results show in any case that for a certain set of subregions of $R+S$, the "regular quadrilaterals," the above stated conclusion is correct. More precisely, these "regular quadrilaterals" are defined by:

Let $S_{1}$ and $S_{2}$ be segments of curves of $S$ having the property that they are nowhere tangent to a characteristic of (2) and being so related that those characteristics of (2) emanating from $S_{1}$ pass out of $R$ on $S_{2}$ and conversely. Here $S_{1}$ signifies that one of the pair of segments across which these characteristics cross into $R$ (referring to (1)). That closed simply-connected subregion of $R+S$ bounded by $S_{1}, S_{2}$, and the two characteristics of (2) joining their endpoints is a "regular quadrilateral." [Thus in our problem $R$ cannot be decomposed as a union of regular quadrilaterals.]

Levinson's result then reads (in our notation):

TheOREM. In a regular quadrilateral we may write

$$
v(x, y, \epsilon)=z(x, y, \epsilon)-w(x, y, \epsilon)
$$

where

$$
w=O\left(\epsilon^{1 / 2}\right)
$$

as $\epsilon \rightarrow+0$

uniformly in the quadrilateral and $w=0$ on $S_{1}$ and $S_{2}$, and where $z(x, y, \epsilon)$ has near and on $S_{2}$ the form $e^{-\theta(x, y) / e} h(x, y)$. Here $g=0$ on $S_{2}$ and $g>0$ off of $S_{2}$ and both $g$ and $h$ are of class $C^{2}$; moreover, at points of the quadrilateral where the above representation is not valid

$$
z=O\left(e^{-\delta / \epsilon}\right) \quad \text { uniformly as } \epsilon \rightarrow+0
$$

for a fixed positive $\delta$.

Therefore it will suffice for us to prove that the stated result holds for a second set of subregions of $R+S$, the "regular triangles," these being defined as follows: 
Let $S_{0}$ be a closed segment of a curve of $S$ having the property that it is nowhere tangent to a characteristic of (2) and such that those characteristics emanating from $S_{0}$ enter into and remain in $R$ (referring to (1)), where they approach one of the $P_{i}$. That simplyconnected subregion of $R+S$ traced out by the characteristics emanating from $S_{0}$ is a "regular triangle."

Moreover to show that, on any regular triangle $T, v(x, y, \epsilon)$ approaches zero as $\epsilon \rightarrow+0$ it will clearly be sufficient to show that $v(x, y, \epsilon)$ approaches zero as $\epsilon \rightarrow+0$ on all subregions $G$ of the following type:

$G$ is a simply-connected subregion of $T$ which is bounded by $S_{0}$, by an orthogonal trajectory to those characteristics of (2) lying in $T$ which intersects the two characteristics making up the "sides" of $T$ but does not intersect $S_{0}$, and by the requisite portions of the "sides" of $T$.

We note, too, that the nontangency condition and the stability of the attractor allow us to consider two other triangles $T_{1}, T_{2}$ such that $T_{2} \supset T_{1} \supset T$ and corresponding subregions $G_{1}, G_{2}$ defined analogously to $G$ (the orthogonal trajectory boundary for $G_{1}$ is taken to be a portion of that for $G_{2}$ and it lies "nearer" to the attractor than does that for $G$, so that $G_{2} \supset G_{1} \supset G$ ). We shall find it convenient to introduce characteristic coordinates on $G_{2}$. Levinson [3] has shown that there are $C^{6}$ functions $\sigma(x, y), \tau(x, y)$ satisfying $A \sigma_{x}+B \sigma_{y}=0, B \tau_{x}-A \tau_{y}=0$ on a region such as $G_{2}$, such that: $\partial(\sigma, \tau) / \partial(x, y) \neq 0$; along characteristics of (2), $\sigma=$ constant; and the curvilinear coordinates $(\sigma, \tau)$ are orthogonal. In addition $A \tau_{x}+B \tau_{y}<0$ if $\tau$ is taken as increasing toward the singularity, as we shall do. We denote the values of $\sigma$ on the characteristic boundaries of $G_{2}$ by $\tilde{\sigma}_{1}$ and $\tilde{\sigma}_{2}$ while those values on the characteristic boundaries of $G_{1}$ are denoted by $\sigma_{1}$ and $\sigma_{2}$ (ordering so that $\tilde{\sigma}_{2}>\sigma_{2}>\sigma_{1}>\tilde{\sigma}_{1}$ ).

The proof proceeds in the following manner: Following a technique used by Kamenomostskaya [2] and Aronson [1], we exhibit functions $W_{1}$ and $W_{2}$ defined on $G_{1}$ which consist of "boundary layer" terms alone, except for terms which are $O\left(\epsilon^{1 / 2}\right)$, and which satisfy

$$
L W_{1}=L v \quad L W_{2}=-L v=L(-v),
$$

where we recall

$$
v=U-u \text {. }
$$

These functions are so chosen that $W_{1}, W_{2}>|v|$ on the boundary of $G_{1}$ for sufficiently small $\epsilon>0$. Use of the maximum principle then implies that for such $\epsilon, W_{1}>v$ and $W_{2}>-v$ throughout $G_{1}$. Finally, in- 
spection of $W_{1}$ and $W_{2}$ shows that they are uniformly $O\left(\epsilon^{1 / 2}\right)$ on $G$, and this yields the desired result.

Now in the preceding outline of the proof, "boundary layer" terms denote functions $H(x, y, \epsilon)$ of the following type:

1. In a neighborhood of a portion of the boundary of the region, $H(x, y, \epsilon)$ is of the form $e^{-o(x, y) / e^{m}} h(x, y)$, where $m$ is a positive constant and where $g, h$ are of class $C^{2}, g$ being positive except on this portion of the boundary. Moreover $H$ is of class $C^{2}$ throughout the entire region and $H$ is uniformly $o(1)$ as $\epsilon \rightarrow+0$, except in the boundary neighborhood.

2. $L H=o(1)$ as $\epsilon \rightarrow+0$, uniformly in the entire region.

From direct substitution it is readily seen that only for $m=1 / 2$ and $m=1$ does the second condition give rise to as few as two equations which the functions $g$ and $h$ must satisfy. For these values the equations to be satisfied are:

$$
\begin{aligned}
& m=1: \quad g_{x}^{2}+g_{y}^{2}-A g_{x}-B g_{y}=0, \\
& \left(A-2 g_{x}\right) h x+\left(B-2 g_{y}\right) h y+(C-\Delta g) h=0, \\
& \text { (3) } \quad m=1 / 2: A g_{x}+B g_{y}=0, A h_{x}+B h_{y}+\left(C+g_{x}^{2}+g_{y}^{2}\right) h=0 \text {, } \\
& \text { or, equivalently, } \\
& g_{\tau}=0,\left(A \tau_{x}+B \tau_{y}\right) h_{\tau}+\left[C+g_{o}^{2}\left(\sigma_{x}^{2}+\sigma_{y}^{2}\right)\right] h=0 .
\end{aligned}
$$

The case $m=1$ was considered by Levinson in his proof of the theorem stated previously. From his work it follows that a boundary layer term having the indicated exponential form $(m=1)$ near and on the orthogonal trajectory boundary of $G_{2}$ can be constructed, where the value of $h(x, y)$ can be specified on this boundary in any $C^{2}$ manner so long as it vanishes near the end points of this boundary. Indeed it is further true that

$$
L H=O(\epsilon)
$$

and that except for the boundary neighborhood involved $H$ is uniformly $O\left(e^{-\delta / e}\right)$ where $\delta$ is a fixed positive constant. For the case $m=1 / 2$, on the other hand, it follows from (3) that $g$ must simply be a function which is constant on each characteristic of (2), so that to obtain a "boundary layer" term by this scheme we require a characteristic boundary (that is, we can only require $g=0$ on a boundary which is characteristic if we are to retain a bona fide boundary layer form). Moreover since $h$ satisfies a linear equation (cf. (3)) whose characteristics coincide with those of (2) we may readily specify this function throughout $G_{1}$ as a solution to an initial value problem. It 
follows that in this case we can obtain a boundary layer term having the indicated exponential form throughout the region $G_{1}$.

We now consider a pair of functions $W_{1}$ and $W_{2}$ defined on $G_{1}$ and having the form:

$$
\begin{aligned}
W_{i}(\sigma, \tau, \epsilon)= & H^{(0)}(\sigma, \tau, \epsilon)+h^{(1)}(\sigma, \tau)\left[e^{-k\left(\sigma-\sigma_{1}\right) / e^{1 / 2}}+e^{-k\left(\sigma_{2}-\sigma\right) / e^{1 / 2}}\right] \\
& +\epsilon^{1 / 2} Z_{i},
\end{aligned}
$$

In the above expression $H^{(0)}(\sigma, \tau, \epsilon)$ is chosen according to Levinson's method for the case $m=1$ to be a boundary layer term for the region $G_{2}$ corresponding to the following values on the orthogonal trajectory boundary (a curve $\tau=$ constant) of $G_{2}$

$$
H^{(0)}=\left\{\begin{array}{l}
M+1, \quad \sigma_{1}<\sigma<\sigma_{2}, \\
0, \tilde{\sigma}_{1}<\sigma<\frac{\tilde{\sigma}_{1}+\sigma_{1}}{2}, \tilde{\sigma}_{2}>\sigma>\frac{\tilde{\sigma}_{2}+\sigma_{2}}{2}, \\
\text { a positive } C^{2} \text { "tieup" function } \\
\frac{\tilde{\sigma}_{1}+\sigma_{1}}{2} \leqq \sigma \leqq \sigma_{1}, \\
\frac{\tilde{\sigma}_{2}+\sigma_{2}}{2} \geqq \sigma \geqq \sigma_{2}
\end{array}\right.
$$

[ $M$ is a uniform bound for $v(x, y, \epsilon)$ on $G_{2}$-use of the maximum principle extended to the inhomogeneous case [3] shows there is a uniform bound for $u(x, y, \epsilon)]$. As for $h^{(1)}(\sigma, \tau)$, we choose it to be that solution of (cf. (3))

$$
\left(A \tau_{x}+B \tau_{y}\right) h_{\tau}+\left[C+k^{2}\left(\sigma_{x}^{2}+\sigma_{y}^{2}\right)\right] h=0
$$

which on the segment of $S$ bounding $G_{1}$ takes on the value $M+1$, while $k$ is a positive constant required to be sufficiently large so that the coefficient of $h$ in (6) is positive throughout $G_{1}$. Thus $h^{(1)}(\sigma, \tau)$ is defined and $h^{(1)} \geqq M+1$ throughout $G_{1}$. Finally, we choose the $Z_{i}$ so that

$$
L W_{1}=L v=\epsilon \Delta U, \quad L W_{2}=L(-v)=-\epsilon \Delta U,
$$

and $Z_{1}$ and $Z_{2}$ vanish on the entire boundary of $G_{1}$. Thus the $Z_{i}$ must solve the homogeneous boundary value problem on $G_{1}$ for

$$
\begin{aligned}
\epsilon^{1 / 2} L Z_{i}= & \epsilon^{1 / 2}\left\{\left[2 k h_{\sigma}^{(1)}\left(\sigma_{x}^{2}+\sigma_{y}^{2}\right)+k h^{(1)} \Delta \sigma\right]\right. \\
& \left.\cdot\left(e^{-k\left(\sigma-\sigma_{1}\right) / \epsilon^{1 / 2}}-e^{-k\left(\sigma_{2}-\sigma\right) / \epsilon^{1 / 2}}\right)\right\} \\
& +\epsilon\left\{\left[h_{\sigma \sigma}^{(1)}\left(\sigma_{x}^{2}+\sigma_{y}^{2}\right)+h_{\tau \tau}^{(1)}\left(\tau_{x}^{2}+\tau_{y}^{2}\right)\right.\right. \\
& \left.\left.+h_{\sigma}^{(1)} \Delta \sigma+h_{\tau}^{(1)} \Delta \tau\right]\left(e^{-k\left(\sigma-\sigma_{1}\right) / \epsilon^{1 / 2}}-e^{-k\left(\sigma_{2}-\sigma\right) / \epsilon^{1 / 2}}\right)\right\} \\
& -L H^{(0)}+\epsilon(-1)^{i+1} \Delta U, \quad i=1,2 .
\end{aligned}
$$


(It follows from results of Lichtenstein that $C^{2}$ solutions to these boundary value problems exist [4].) It therefore follows from the inhomogeneous case maximum principle [3] that $Z_{i}=O(1)$ as $\epsilon \rightarrow+0$, uniformly on $G_{1}$ [recalling that $L H^{(0)}=O(\epsilon)$ ].

Now examination of the functions $W_{1}$ and $W_{2}$ indicates that in $G$ $W_{i}=O\left(\epsilon^{1 / 2}\right)$ uniformly as $\epsilon \rightarrow+0$. Moreover $W_{1}-v$ and $W_{2}+v$ satisfy the homogeneous equation $L u=0$, while investigation of the boundary values shows that, for sufficiently small $\epsilon, W_{1}-v$ and $W_{2}+v$ are positive throughout the boundary of $G_{1}$. Thus the maximum principle shows that for these $\epsilon, W_{1}-v \geqq 0$ and $W_{2}+v \geqq 0$ throughout $G_{1}$. In particular,

$$
|v| \leqq W_{1}+W_{2}=O\left(\epsilon^{1 / 2}\right) \quad \text { throughout } G,
$$

which completes the proof.

\section{BIBLIOGRAPHY}

1. D. Aronson, Linear parabolic equations containing small parameters, to appear in Journal of Rational Mechanics and Analysis.

2. S. L. Kamenomostskaya, On equations of elliptic and parabolic type with a small parameter in the highest derivative, Mat. Sbornik N.S. vol. 31(73) (1952) pp. 703-708. (Russian).

3. N. Levinson, The first boundary value problem for $\epsilon \Delta u+A(x, y) u_{x}+B(x, y) u_{y}$ $+C(x, y) u=D(x, y)$ for small $\epsilon$, Ann. of Math. vol. 51 (1950) pp. 428-445.

4. L. Lichtenstein, Randwertaufgaben der Theorie der linearen partiellen Differentialgleichungen zweiter Ordnung von elliptischen Typus, J. Reine Angew. Math. vol. 142 (1913) pp. 1-40.

Massachusetrs Institute of Technology 